\title{
A Case of Fever of Unknown Origin: Co-existence of Kikuchi-Fujimoto Disease and Acute Disseminated Encephalomyelitis (ADEM)
}

\author{
Vildan Avkan-Oguz ${ }^{1}$, Nur Yapar ${ }^{1}$, Serkan Ozakbas ${ }^{2}$, Kubra Demir-Onder ${ }^{1}$, Eray Aktas ${ }^{1}$, \\ Sema Alp-Cavus ${ }^{1}$, Sermin Ozkal ${ }^{3}$, Suleyman $\mathrm{Men}^{4}$ and Aydanur Kargi ${ }^{3}$
}

\begin{abstract}
It is believed that viral infections and the hyperimmune reaction due to these infections are involved in the etiology of Kikuchi-Fujimoto Disease (KFD), a rare cause of fever of unknown origin. Axillary lymphadenopathy and neurologic involvement are rare in KFD. We present a patient diagnosed with KFD histopathologically during an investigation of the origin of fever and axillary lymphadenopathy. Subsequently, incidental sinus aspergilloma was diagnosed radiologically in the patient and acute disseminated encephalitis developed during follow-up. This report aims to draw attention to the co-existence of KFD and Acute Disseminated Encephalomyelitis, two diseases of which the origins are not clear.
\end{abstract}

Key words: fever of unknown origin (FUO), Kikuchi-Fujimoto disease, histiocytic necrotizing lymphadenitis, acute disseminated encephalomyelitis (ADEM)

(Inter Med 49: 1823-1826, 2010)

(DOI: 10.2169/internalmedicine.49.3633)

\section{Introduction}

It is believed that viral infections and the hyperimmune reaction that occurs due to these infections are involved in the etiology of Kikuchi-Fujimoto Disease (KFD), which is a rare cause of fever of unknown origin (FUO). It is reported that neurological manifestations including aseptic meningitis, cerebellar ataxia, mononeuritis multiplex, hemiparesis and brachial neuritis may be detected during the course of the disease (1). However, to date, acute disseminated encephalomyelitis (ADEM) following KFD has not been reported. ADEM is classified under demyelinating diseases of the central nervous system with a monophasic course and its diagnosis can only be made after all of the other possible diseases are ruled out. Although the etiology of both ADEM and KFD still remains obscure, it is believed that previous infections may play a role in their pathogenesis. The present patient was diagnosed with KFD histopathologically during the investigation aimed at discovering the origin of her fever and lymphadenopathy. Subsequently, she was diagnosed with incidental sinus aspergilloma radiologically and developed ADEM during her follow-up. The present report aims to draw attention to the co-existence of two diseases of unknown origin, KFD and ADEM, which probably share the same etiology.

\section{Case Report}

A 36-year-old woman applied to our clinic with complaints including fever $\left(39.5^{\circ} \mathrm{C}\right)$, lack of appetite, vomiting and diarrhea, which had been present for about one month. She stated that all of these complaints had appeared 10 days after the formation of a painful swelling under her axillary region. During the 40 days that she had spent without receiving hospital treatment, the patient had used amoxicillin, clavulanic acid, levofloxacin and third generation cephalosporins on an irregular basis. She had no history of close

\footnotetext{
${ }^{1}$ Department of Infectious Diseases and Clinical Microbiology, Dokuz Eylul University School of Medicine, Izmir, Turkey, ${ }^{2}$ Department of Neurology, Dokuz Eylul University School of Medicine, Izmir, Turkey, ${ }^{3}$ Department of Pathology, Dokuz Eylul University School of Medicine, Izmir, Turkey and ${ }^{4}$ Department of Radiology, Dokuz Eylul University School of Medicine, Izmir, Turkey

Received for publication March 8, 2010; Accepted for publication May 12, 2010

Correspondence to Dr. Vildan Avkan-Oguz, vildan.oguz@gmail.com
} 
Table 1. The Laboratory Findings of the Patient

\begin{tabular}{|l|c|c|l|}
\hline Laboratory findings & In admission & At the discharge & Reference range \\
\hline Leukocyte count & 3400 & 7400 & $4000-10300 / \mathrm{mm}^{3}$ \\
Hemoglobin & 10.5 & 10,0 & $12-16 \mathrm{gr} / \mathrm{dL}$ \\
Platelet & 203000 & 156000 & $156000-373 \mathrm{000} / \mu \mathrm{L}$ \\
C-reactive protein & 144 & 21 & $0.1-8.2 \mathrm{mg} / \mathrm{L}$ \\
Erythrocyte sedimentation rate & 55 & 11 & $0-20 \mathrm{~mm} / \mathrm{hr}$ \\
Aspartate aminotransferase & 84 & 35 & $5-34 \mathrm{U} / \mathrm{L}$ \\
Alanine aminotransferase & 52 & 54 & $0-55 \mathrm{U} / \mathrm{L}$ \\
Lactic dehydrogenase & 185 & 215 & $125-243 \mathrm{U} / \mathrm{L}$ \\
Creatinin & 0.79 & 0,65 & $0.6-1.1 \mathrm{mg} / \mathrm{dL}$ \\
Albumin & 2.7 & 4,3 & $3.5-5 \mathrm{~g} / \mathrm{dL}$ \\
Cerebrospinal fluid: & 49 & & \\
Glucose & 61 & - & $>40 \mathrm{mg} / \mathrm{dL}$ \\
$\quad$ Protein & 40 neutrophilic & - & $15-45 \mathrm{mg} / \mathrm{dL}$ \\
Cell count & 366 & - & $6.3-33.5 \mathrm{mg} / \mathrm{L}$ \\
IgG & 718 & - & $100-300 \mathrm{mg} / \mathrm{L}$ \\
Albumin & 0.66 & - & $0.3-0.7$ \\
IgG index & &
\end{tabular}
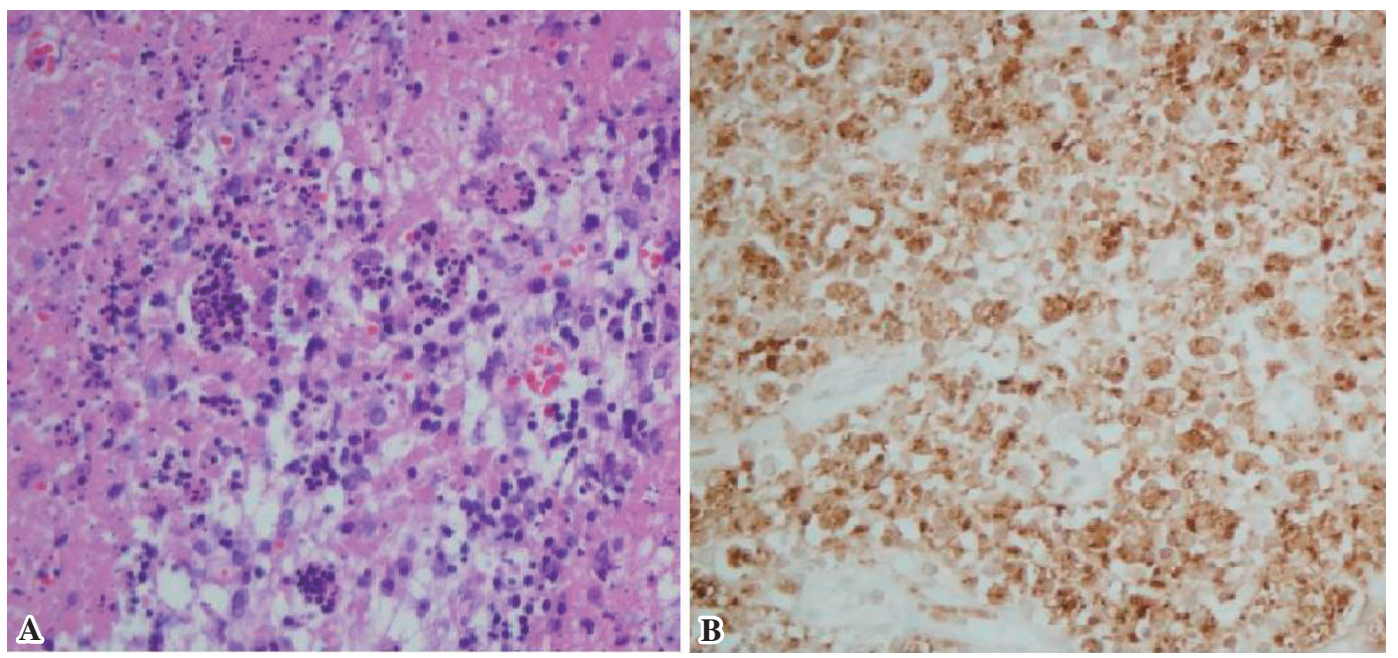

Figure 1. A: Karryorrhectic debris, histiocytes, and lymphoid cells are seen in the necrotic areas. $\times 400$, Hematoxylin and Eosin staining. B: Numerous MPO positive histiocytes are revealed in the necrotic area. $\times 400$, MPO

contact with pets; she did not travel often and similar complaints were not present in her social circle. According to the patient's physical examination, her general health status was good; body temperature, pulse, and blood pressure were $39^{\circ} \mathrm{C}, 84 / \mathrm{min}$ and $100 / 60 \mathrm{mmHg}$, respectively. She was alert, oriented and co-operative. On the right side of the axillary region, markedly mobile, painless lymphadenopathies (LAPs), of which the largest was $3 \times 2 \mathrm{~cm}$ in size, were present. Cardiac examination revealed a $2 / 6$ systolic murmur. Organomegaly and LAPs in the inguinal region were not palpated. Examination of the respiratory and neurologic systems revealed normal findings.

Laboratory examinations revealed the following findings (Table 1). No positive results that could explain high body temperature were found during the serological tests for Epstein Barr virus (EBV), cytomegalovirus (CMV), hepatitis A, B, and C, Toxoplasma, Brucella, and Salmonella spp. Blood cultures were incubated for 6 to 8 weeks and Bru- cella spp did not grow in them. Vegetation was not detected by transthoracic echocardiogram; her body temperature could not be lowered by routine anti-inflammatory drugs and intravenous paracetamol. Rheumatologic markers (ferritin, anti-nuclear antibody, anti-neutrophil cytoplasmic antibody, anti-cyclic citrullinated peptide antibodies, extractable nuclear antigen antibody panel, anti-double stranded DNA, rheumatoid factor) were negative.

On the 7th day of hospitalization, an excision biopsy of axillary lymph node was performed. Microscopic examination revealed effacement of nodal architecture by patchy necrosis. The necrotic areas included nuclear dust and cellular debris, but not polymorphonuclear neutrophilic leucocyte. These necrotic areas were surrounded by histiocytes which express myeloperoxidase (MPO) and CD68 (Fig. 1A, 1B). Residual germinal centers and activated lymphocytes were also found in the paracortical areas. Lymphoid cells showed mainly T-cell phenotype. Only a low number of CD20 posi- 


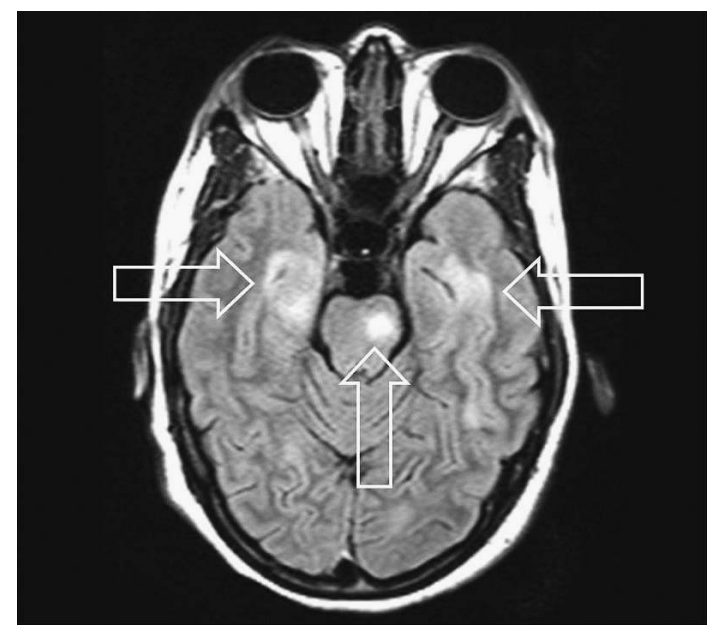

Figure 2. Transverse FLAIR-weighted image shows areas of high signal in the medial temporal lobes and pons (arrows).

tive B-cells were found.

Thorax and abdomen computerized tomography (CT) scan revealed splenomegaly; lymph nodes were predominantly located on the right side of the bilateral axillary region, in addition to lymph nodes in the paracaval, paraaortic and bilateral internal iliac chains, of which the largest was $1.5 \mathrm{~cm}$ in diameter. Thus, one of the lymph nodes located in the right axillary region was removed and sent to pathology for analysis. Since subtle vasculitic alterations were determined by ophthalmic fundus examination, brain magnetic resonance imaging (MRI) was scheduled. Before MRI could be performed, the patient had a generalized tonic clonic seizure on the 13th day of hospitalization. After the seizure, the patient was no longer oriented and cooperative. Urinary incontinence developed and the patient's agitation could not be controlled by sedative drugs. CT was urgently performed. Since CT exam revealed subtle hypodensity in the temporal region, acyclovir treatment was started because it was not possible to rule out encephalitis. After the patient's status was stabilized, MRI was performed under sedation and it revealed aspergilloma in the sphenoid sinus and transverse FLAIR-weighted image showed areas of high signal in the medial temporal lobes and pons. These lesions, of which the diffusion was not consistent with lymphoma, suggested the presence of limbic encephalitis, encephalitis of viral origin, central nervous system tuberculosis and ADEM (Fig. 2).

Lumbar puncture was performed. The CSF sample was submitted for bacteriologic, mycologic and mycobacteriologic examination and evaluated with serological and pathological tests (Table 1). Growth was not observed in the non-specific or mycotic cultures of the CSF sample. ZiehlNielseen staining and culture were negative. Additionally, the Herpes simplex virus (HSV), Varicella zoster virus (VZV), Enterovirus, Adenovirus and M. tuberculosis polymerase chain reaction (PCR) tests were negative. Oligoclonal band was not detected in the CSF sample. CSF protein level was above the upper limit and pleocytosis was observed.

At the end of the second week of hospitalization, the fre- quency and duration of the patient's apnea episodes increased and her general status worsened during these episodes. Since ADEM was highly suspected, prednisolone treatment ( $1 \mathrm{~g} /$ day iv) and follow-up in the intensive care unit were started. Following 24 hours of steroid treatment, the patient demonstrated a significant clinical response, her level of awareness improved and her body temperature returned to normal. Acyclovir treatment was prolonged to 10 days and pulse steroid treatment was continued for 10 days. Neurological status dramatically improved from the third day of steroid treatment. After the stabilization of the patient's general status, an endoscopic sinus intervention was performed due to the incidental sphenoidal sinus aspergilloma and a 2-week long antifungal treatment was started. Aspergillus fumigatus grew from the culture of the sample taken during the endoscopy. The steroid treatment was gradually tapered and terminated after 3 months. Axillary lymphadenopathies and splenomegaly disappeared during the follow-up. MRI investigation of the brain revealed that the previous abnormalities were no longer present. When this finding combined with significant response to steroid treatment diagnosis of clinical status was confirmed as ADEM. Treatment has been discontinued and the patient has been followed up for a year; no complications have been observed to date.

\section{Discussion}

Kikuchi-Fujimoto Disease, which was first described in Japan in 1972 and is mostly seen in the Far East, has begun to be reported in the other parts of the world recently (1-3). Although the etiology of the disease remains unknown, infectious or autoimmune pathogenesis is held responsible. The factors which likely result in the infection include EBV, CMV, Human herpes virus-6, VZV, Human immunodeficiency virus, rubella, measles, coronavirus, Coxsackie virus, hepatitis A and B, Yersinia enterocolitica, toxoplasma, influenza viruses, streptococci, leptospira and chlamydia. It has also been reported that the disease may occur after rabies, diphtheria-tetanus, poliomyelitis, hepatitis B and influenza vaccines are injected (4). The disease may initially be mistaken for various benign and malignant diseases, such as tuberculosis, lymphoma, systemic lupus erythematosus (SLE) and FUO. In the studies on FUO patients conducted in Turkey, KFD has not been mentioned as a cause of fever $(5,6)$. According to the English language literature, KFD is a rare cause of fever of unknown origin (7) and cervical LAP is seen in $70-98 \%$ of KFD cases while the rate of axillary LAP is lower (8). The co-existence of axillary lympadenopathy and KFD is the least common clinical picture of KFD, which is usually mistaken for lymphoma. Thus, KFD should be included in the differential diagnosis in patients with axillary LAPs. The definitive diagnosis can be based on histopathological examination. In the present case, the histopathological features were characterized by presence of necrosis with histiocytes and cellular debris. We considered 
a necrotizing type of Kikuchi-Fujimoto disease. Three different histological subtypes of this disease have been defined: proliferative, necrotizing and xanthomatous $(9,10)$.

Neurological involvement is also rarely reported in studies investigating patients diagnosed with KFD (11). In their study evaluating 244 KFD patients, Kucukardali et al reported the rate of neurologic involvement findings including aseptic meningitis, mononeuritis multiplex, hemiparesis, brachial neuritis and photophobia as 5\% (1). Since the present patient was diagnosed with KFD histopathologically but generalized tonic clonic seizure occurred during the continuing examinations aimed at determining the etiology of the disease, the differential diagnosis had to be reconsidered. Because viral encephalitis could not be ruled out, antiviral treatment was started. However, the treatment of the patient was changed due to the fact that central nervous system involvement could not be explained by encephalitis or KFD and the lesions detected by MRI were consistent with ADEM. In general, MRI findings include multifocal areas of high signal intensity in the supratentorial white matter, brain stem and cerebellum. The deep gray matter is frequently involved as well. After the initiation of corticosteroid therapy, follow-up imaging demonstrates decrease in size and number of the lesions (12). According to the consensus definition for ADEM reported by Sonneville et al, the present patient's status fulfilled all of the diagnostic criteria of ADEM with acute clinical attack of inflammatory disease of CNS, affected multifocal areas of CNS, polysymptomatic presentation including encephalopathy, and clear improvement on clinical and neuroradiological measures (4). It was advantageous that corticosteroid treatment was used for the treatment of both KFD and ADEM. The incidental aspergilloma that was detected by radiological imaging during the highdose corticosteroid therapy, which was required for the treatment of ADEM, could not be overlooked. Antifungal treatment was started along with the corticosteroid therapy. Due to the lack of knowledge related to the co-existence of these two diseases and the consequent impairment of immune response, antifungal therapy was used in addition to endoscopic sinus intervention. No bacterial or viral infections were detected during the available tests except HHV-6 of which serological tests could not be performed. It was striking that allergic inflammatory or autoimmune response played a role in the etiology of all three clinical manifestations (aspergilloma, ADEM and KFD) (2, 4, 13, 14). A review of the accessible literature did not show any reports of the co-existence of aspergilloma and KFD or co-existence of aspergilloma and concomitant $\mathrm{KFD}$ and $\operatorname{ADEM}(1,4,15)$. We supposed that, instead of infectious etiology, the clinical picture in this first case of ADEM and KFD co-existence might have resulted from the patient's autoimmune response or immune reconstitution (IRIS).

As a result, unexpected clinical findings may be encountered during the follow-up of patients with diseases of unknown etiology such as KFD. In such patients, the existence of neurological involvement particularly worsens the clinical status and contributes to mortality. Thus, ADEM, which can reach a mortality rate of $25 \%$, should be considered in the differential diagnosis when central nervous system involvement is detected during the follow-up of a patient with KFD.

\section{References}

1. Kucukardali Y, Solmazgul E, Kunter E, Oncul O, Yildirim S, Kaplan M. Kikuchi-Fujimoto Disease: analysis of 244 cases. Clin Rheumatol 26: 50-54, 2007.

2. Baumgarten BJ, Helling ER. Kikuchi's disease: A case report and review of the literature. Ear Nose Throat J 81: 331-335, 2002.

3. Adhikari RC, Sayami G, Lee MC, Basnet RB, Shrestha PK, Shrestha HG. Kikuchi-Fujimoto disease in Napal: a study of 6 cases. Arch Pathol Lab Med 127: 1345-1348, 2003.

4. Sonneville R, Klein I, De Broucher T, Wolff M. Post-infectious encephalitis in adults: Diagnosis and management. J Infect 58: 321-328, 2009.

5. Tabak F, Mert A, Celik AD, et al. Fever of unknown origin in Turkey. Infection 31: 417-420, 2003.

6. Sipahi OR, Senol S, Arsu G, et al. Pooled analysis of 857 published adult fever of unknown origin cases in Turkey between 1990-2006. Med Sci Monit 13: CR318-CR322, 2007.

7. Cunha BA, Mickail N, Durie N, Pherez FM, Strollo S. Fever of unknown origin (FUO) caused by Kikuchi's disease mimicking lymphoma. Heart Lung 38: 450-456, 2009.

8. Kovacs S, Friedman PD, Kuehn A. Unilateral axillary adenopathy caused by Kikuchi-Fujimoto disease. Breast J 12: 77-79, 2006.

9. Hrycek A, Cieslik P, Szkrobka W, Pajak J. Kikuchi-Fujimoto disease: a case report. Rheumatol Int 26: 179-181, 2005.

10. Bosch X, Guilabert A. Kikuchi-Fujimoto disease. OJRD (www. OJRD.com/content/1/1/18).

11. Moon JS, $11 \mathrm{Kim}$ G, Koo YH, et al. Kinetic tremor and cerebellar ataxia as initial manifestations of Kikuchi-Fujimoto's disease. J Neurol Sci 277: 181-183, 2009.

12. Nusbaum AO, Rapalino O, Fung KM, et al. White matter diseases and inherited metabolic disorders. In: Magnetic Resonance Imaging of the Brain and Spine. Fourth, Ed. Volume one. Atlas SW, Ed. Wolters Kluwer/Lippincott Williams and Wilkins, Philedelphia, 2009: 343-444.

13. Curone M, D'Amico D, Maccagnano E, Bussone G. Fatal Aspergillus brain abscess in immunocompetent patient. Neurol Sci 30: 233-235, 2009.

14. Lin DY, Villegas MS, Tan PL, Wang S, Shek LP. Severe Kikuchi's disease responsive to immune modulation. Singapore Med J 51: e18-e21, 2010.

15. Noorbakhsh F, Johnson RT, Emery D, Power C. Acute disseminated encephalomyelitis: clinical and pathogenesis features. Neurol Clin 26: 759-780, 2008.

(C) 2010 The Japanese Society of Internal Medicine http://www.naika.or.jp/imindex.html 